\title{
Focus prominence and tonal alignment in Athenian and Cypriot Greek
}

Charalambos Themistocleous, Stelios Kyriacou

Phonetics Laboratory, University of Athens, Greece

https://doi.org/10.36505/ExLing-2010/03/0046/000166

\begin{abstract}
This study examines the effects of focus prominence production on tonal alignment in Athenian Greek and Cypriot Greek. Information focus has been elicited by using a Wh-Question in utterances with varying number of syllable constituents - from twelve syllables to eighteen - following the constituent under focus prominence. The results indicate significant effects of speech variety on the alignment of tonal targets; utterance length on the other hand showed poor effects on tonal alignment.
\end{abstract}

Key words: focus, intonation, Athenian Greek, Cypriot Greek.

\section{Introduction}

This study examines the effects of focus prominence on tonal alignment, in utterances with varying length, in Athenian Greek (henceforth AG) and Cypriot Greek (henceforth CG). Focus is manifested in speech as a post-lexical prominence realized intonationally by the exploitation of nuclear pitch accents and phrase accents (c.f. Botinis, Bannert, Tatham, 2000). Importantly, the exact alignment of tonal targets comprising the nuclear pitch accents plays a significant role in the phonetic realization of nuclear pitch accents (c.f. Bruce, 1977). Two main questions are addressed: (a) what is the effect of utterance length on the alignment of nuclear pitch accents, and (b) how do the two varieties of Greek differ in the tonal alignment of nuclear pitch accents.

\section{Methodology}

Information focus on a prepositional phrase constituent functioning as a verbal compliment has been elicited by the use of Wh-Questions, specific for each language variety. Specifically, the constituent under focus prominence was produced in three different contexts which varied in the number of syllables following the constituent under focus prominence (see Table 1). The materials were produced by ten educated speakers in total, one male and four female speakers for each variety. AG speakers were brought up in Athens and CG speakers in the main urban areas of Cyprus. All speakers were in their early twenties. All ten speakers produced focus prominence in 3 sentence carriers $x 5$ repetitions, a total of 150 utterances.

ExLing 2010: Proceedings of 3rd Tutorial and Research Workshop on Experimental Linguistics, 25-27 August, Athens, Greece 
Table 1. The Cypriot Greek material with varying number of syllables following the constituent with focus prominence.

\begin{tabular}{|c|c|}
\hline Utterances & Syllables \\
\hline 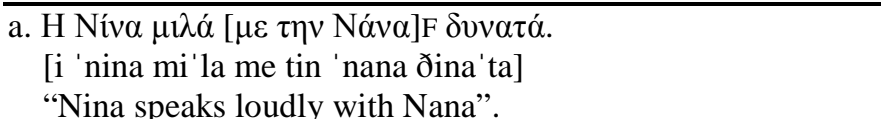 & [12] Twelve \\
\hline 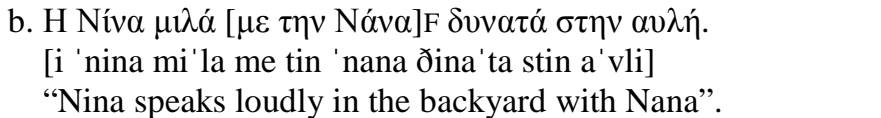 & [15] Fifteen \\
\hline 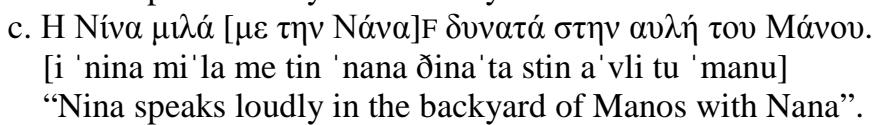 & [18] Eighteen \\
\hline
\end{tabular}

The utterances were recorded using Praat, with $22050 \mathrm{~Hz}$ sampling frequency. The test words were manually segmented and labelled, by using simultaneous inspections of waveforms and wide-band spectrograms following the segmentation criteria of Peterson \& Lehiste (1960). The following measurements were obtained from the data (see Figure 1): the distance of local minimum (L) and local maximum (H) (a) from the onset of the first syllable of the word "'nana" $\{\mathrm{n}\}$, (b) from the beginning of the nucleus of the first syllable of the word /'nana/ $\{\mathrm{v}\}$ and (c) from the right edge of the word /'nana/ $\{\mathrm{we}\}$. The distance of turning point (TP) from the $\{$ we $\}$ was also measured. A two way ANOVA (language variety $\mathrm{x}$ utterance category) was performed for each variable.

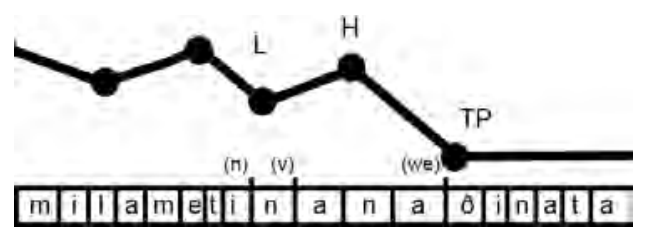

Figure 1. Stylized contour of the utterance /' mila me ti 'nana ðina'ta/ and its associated segmental string. The dots represent tonal targets while the solid lines the interpolated pitch contours. $\mathrm{L}$ stands for local minimum, $\mathrm{H}$ for local maximum, TP for turning point, $\{\mathrm{n}\}$ for the onset of the syllable (na), $\{\mathrm{v}\}$ for nucleus beginning and $\{$ we $\}$ for word /'nana/ right edge.

\section{Results}

AG and CG productions show apparent similarities in the realization of focus prominence (see Figure 2). A rise and a fall are associated with the stressed syllable of the lexical constituent in focus. The local minimum of the F0 contour aligns at the onset of the stressed syllable, while the local maximum of the F0 contour aligns at the onset of the post-stressed syllable 
(see Figure 2). The turning point (TP) aligns at the coda of the lexical constituent in focus prominence.

(a) Athenian Greek utterances

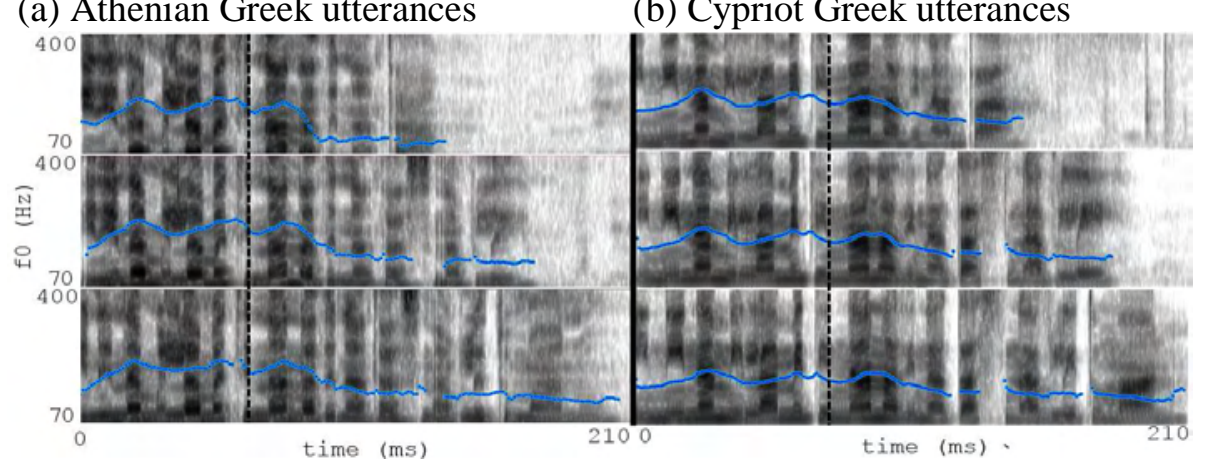

Figure 2. Panel (a) shows the productions of a female AG speaker and Panel (b) shows the productions of a female CG speaker for the utterances a-c. The dashed line indicates the onset of the constituent in focus ['nana].

Language variety however let to different productions in tonal alignment (see Figure 3). ANOVA tests for the effects of variety and utterance type on the distance between $\mathrm{L}$ from the onset of the stressed syllable [na] were significant for variety $(F(1,144)=10.286, p<.05, r=.36)$ and for utterance category $(F(2,144)=3.157, p<.05, r=.2)$. Also, the distance between $\mathrm{H}$ from the onset of [na] was significant for variety $(F(1,144)=15.567$, $p<.0001, r=.39)$ and utterance category $(F(2,144)=6.268, p<.05, r=.3)$. Language variety affected significantly the distance between $\mathrm{L}(F(1,144)=$ $25.622, p<.0001, r=.5)$ and $\mathrm{H}(F(1,144)=28.489, p<.0001, r=.5)$ from the beginning of syllable nucleus $\{\mathrm{v}\}$ and the distance between TP from $\{$ we $\}(F(1,144)=28.489, p<.0001, r=.5)$. All other effects were nonsignificant.
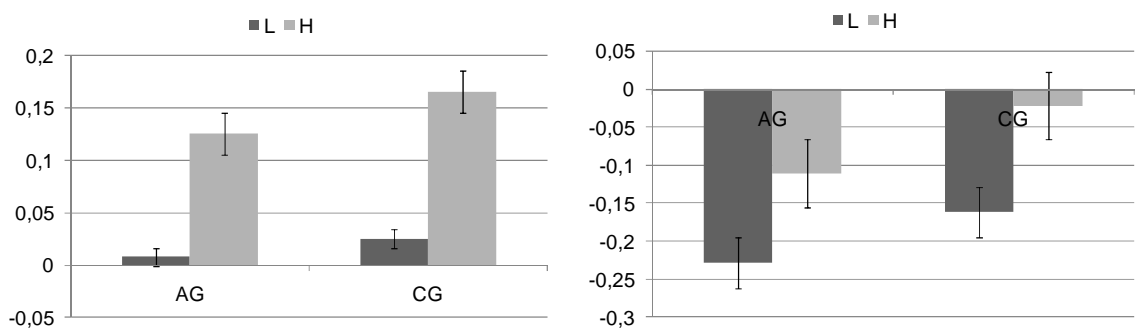

Figure 3. The mean distance in seconds of local minimum (L) and local maximum $(\mathrm{H})$ from the onset of the first syllable of the word "nana" (panel A) and from the nucleus of the first syllable of the word "nana" (panel B) is shown for each variety. Error bars show SE. 


\section{Discussion}

This study examined the phonetic realization of nuclear pitch accents signalling information focus on a prepositional phrase constituent functioning as a verbal compliment. Importantly, both language varieties show apparent similarities in the realization of the nuclear pitch accent; a rise and a fall are associated with the stressed syllable of the lexical constituent in focus. The local minimum of the F0 contour aligns at the onset of the stressed syllable, while the local maximum of the F0 contour aligns at the onset of the post-stressed syllable. A turning point following local maximum aligns at the coda of the final syllable of the lexical constituent in focus prominence and functions as the left anchoring point of a level declining contour that terminates at the utterance's right edge (c.f. Baltazani, Jun, 1999, Botinis et al. 2000).

The results show significant effects of language variety on the alignment of tonal targets of nuclear pitch accent, indicating systematic differences in the phonemic and phonetic realization of nuclear pitch accents in the two language varieties. Both language varieties show structural consistency in the alignment of tonal targets with the segmental string as manifested by the poorly significant effects of utterances' length on tonal alignment. Further research is needed so as to gain specific understanding of the interaction between different focus categories and their phonemic and phonetic manifestation.

\section{Acknowledgements}

The present study has been supported by the University of Athens in the framework of the programme "Kapodistrias", project "Text prosody in Greek". The authors would like to thank the participants of the experiment as well as Antonis Botinis, Marios Fourakis and Spyros Armostis for comments and much useful feedback.

\section{References}

Baltazani, M., Jun S. 1999. Topic and focus intonation in Greek. Proceedings of the XIVth International Congress of Phonetic Sciences, vol. 2, 1305-1308.

Botinis, A., Bannert R., Tatham M. 2000. Contrastive tonal analysis of focus perception in Greek and Swedish. In Botinis, A. (ed.), Intonation: Analysis, modelling and technology. Dordrecht: Kluver Academic Publishers, 97-118.

Bruce, G.. 1977. Swedish word accents in sentence perspective. Lund: Gleerup.

Peterson, G..E., Lehiste, I. 1960. Duration of Syllable Nuclei in English. The Journal of the Acoustical Society of America, 32(6), 693-703. 